\title{
Protocole EMDR individuel pour une utilisation paraprofessionnelle : un essai randomisé contrôlé auprès de premiers intervenants
}

\author{
Ignacio Jarero \\ Carolina Amaya \\ Martha Givaudan \\ Alaide Miranda \\ Fondation latino-américaine et caraïbéenne pour la recherche sur les psychotraumatismes, México, Mexique
}

\begin{abstract}
Le protocole EMDR (désensibilisation et retraitement par les mouvements oculaires) individuel pour une utilisation paraprofessionnelle dans les situations de traumatisme aigu (EMDR-PROPARA) fait partie d'un projet développé à l'initiative du Dr Francine Shapiro. Cet essai clinique randomisé a examiné l'efficacité de ce protocole, administré par des thérapeutes EMDR expérimentés. Trente-neuf premiers intervenants en service actif ayant subi des traumatismes ont été répartis de manière aléatoire en deux groupes et ont reçu des séances de quatre-vingt-dix minutes, soit d'EMDR-PROPARA, soit de thérapie de soutien. Les participants du groupe EMDR-PROPARA ont montré une amélioration immédiatement après le traitement, et leurs scores au Short PTSD Rating Interview (SPRINT), Iors d'un suivi à trois mois, avaient encore baissé. Comparativement, les participants du groupe ayant bénéficié de la thérapie de soutien présentaient une amélioration non significative après le traitement et une augmentation des scores au SPRINT lors du second suivi. La différence significative entre les deux traitements donne un support préliminaire à l'hypothèse de l'efficacité de I'EMDR-PROPARA pour réduire la gravité des symptômes post-traumatiques et favoriser une amélioration subjective globale. Les auteurs recommandent que des études mieux contrôlées soient menées afin d'évaluer plus précisément l'efficacité de cette intervention.
\end{abstract}

Mots-clés : trauma aigu ; EMDR ; intervention psychologique précoce ; premiers intervenants ; soutien par les pairs.

$\mathbf{L}$ a santé mentale et le bien-être des premiers intervenants sont importants pour les individus, leur famille, l'organisation à laquelle ils appartiennent et plus largement, la communauté (Scully, 2001 ; Shakespeare-Finch, 2011). Leurs difficultés psychologiques représentent des coûts personnels et publics importants et peuvent porter atteinte à la qualité des services qu'ils rendent aux victimes et aux patients (Kleim \& Westphal, 2011).

Dans cet article, nous utiliserons le terme " premiers intervenants " pour parler d'un ensemble hétérogène de professionnels rémunérés ou de volontaires qui fournissent des services cruciaux en situation d'urgence (p. ex. le soutien au personnel par les pairs dans les grandes entreprises). Historiquement, les professions exercées par les premiers intervenants comprennent les métiers relatifs à l'application de la loi, les opérateurs des lignes téléphoniques d'urgence, les pompiers, le personnel de recherche et de sauvetage, le personnel ambulancier et les équipes des services d'urgences hospitalières. Ces emplois se caractérisent par des niveaux élevés d'exigence au travail

This article originally appeared as Jarero, I., Amaya, C., Givaudan, M., \& Miranda, A. (2013). EMDR Individual Protocol for Paraprofessional Use: A Randomized Controlled Trial With First Responders. Journal of EMDR Practice and Research, 7(2), 55-64. Translated by François Mousnier-Lompré. 
et par une exposition quotidienne à des facteurs tant physiques que psychologiques de stress traumatique (Penalba, McGuire \& Leite, 2008).

Les recherches ont montré qu'une exposition aux facteurs de stress traumatique augmente la probabilité d'apparition d'un état de stress post-traumatique (ESPT), d'autres troubles psychiatriques et d'un burnout (Haugen, Evces \& Weiss, 2012). Les troubles de type traumatique les plus fréquents chez les premiers intervenants sont l'ESPT, le trouble dépressif majeur et les troubles liés à l'alcool et à la drogue (Benedek, Fullerton \& Ursano, 2007). Les premiers intervenants n’ayant pas été spécifiquement formés aux interventions en cas de catastrophe montraient des taux plus élevés de prévalence de l'ESPT (Guo et coll., 2004).

\section{Clarification des termes dans les situations de trauma aigu}

A. État de stress aigu : événement traumatique aigu qui s'est déroulé sur quelques jours et se traduit par des niveaux importants de groupes de symptômes (hyperactivation, évitement, dissociation et intrusion) qui n'ont pas disparu après au moins quelques jours.

B. Événement récent : événement traumatique qui a eu lieu sur une période passée de deux à trois mois et où le participant a connu une période de sécurité post-traumatique.

C. Réseau de souvenirs d'exposition traumatique cumulative : événements stressants corrélés qui se sont poursuivis sur une période longue (souvent plus de 3 mois) et où le participant n'a pas connu de période de sécurité post-traumatique. Ce genre de situation traumatique peut être rencontré par le personnel des organisations à haut risque (p. ex. le personnel militaire et les premiers intervenants en service actif), par les survivants de catastrophes naturelles ou d'origine humaine, par les immigrants illégaux, les crises géopolitiques, etc.

Pour Jarero et Uribe (2011, 2012), les situations traumatiques aiguës ne sont pas seulement en rapport avec un cadre temporel (jours ou mois), mais également avec une période post-traumatique de sécurité. Ils font valoir que l'absence d'une période post-traumatique de sécurité empêche la consolidation, dans la mémoire, de l'incident critique original parce que le continuum d'événements stressants contenant des informations similaires (p. ex. émotions, sensations physiques) ne laisse pas au souvenir traumatique dépendant d'état (Van der Kolk \& Van der Hart, 1991) le temps de se consolider et de former un ensemble intégré. Le réseau de souvenirs reste ainsi dans un état d'excitation permanent, devenant plus sensible et plus large à chaque nouvel événement stressant dans ce continuum, comme les rides à la surface d'un lac lorsqu'on y jette une pierre, avec le risque d'apparition d'un état de stress post-traumatique (ESPT) et de troubles comorbides s'aggravant avec le nombre d'expositions.

\section{Traitement du traumatisme des premiers intervenants}

Dans les zones ayant peu ou pas de prise en charge psychologique professionnelle des traumas (p. ex. les pays en voie de développement), les premiers intervenants exposés à des traumas directs ou secondaires ou à une fatigue compassionnelle (Figley, 1995) au sein de leur environnement professionnel et qui ne parviennent pas à guérir au fil du temps ou après une intervention de crise, peuvent tirer profit d'un traitement précoce fourni par un pair. Selon Creamer et coll. (2012) :

La justification de ces apports de programmes de soutien par les pairs est souvent de répondre au devoir légal et moral de prendre en charge les employés, ainsi que de surmonter les multiples obstacles s'opposant à une prise en charge standard (stigmatisation, manque de temps, difficulté d'accès aux professionnels, manque de confiance, peur de conséquences sur son emploi). (p. 134)

\section{Les interventions aiguës}

L'intervention précoce est censée empêcher l'apparition ultérieure d'une détresse psychologique ou d'une morbidité psychologique de longue durée (Scully, 2011). On débat beaucoup du type d'intervention à proposer, du moment opportun de ces interventions et des personnes à qui elles devraient s'adresser après une exposition à des événements traumatiques (Roberts, Kitchiner, Kenardy \& Bisson, 2010). Des experts (par exemple, Bisson, Roberts \& Macho, 2003 ; Brewin et al., 2008) estiment que ces interventions doivent se concentrer sur les personnes qui ont le plus de risque de subir des problèmes psychologiques persistants à la suite d'une exposition à des événements potentiellement traumatisants. Plusieurs auteurs emploient un vocabulaire différent pour parler des états de stress aigu et de leur traitement.

Intervention de crise est l'expression employée par Everly et Mitchell (2008) pour décrire un « soin psychologique ou comportemental d'urgence, conçu pour stabiliser d'abord, puis réduire les symptômes 
de détresse ou de dysfonctionnement, de manière à amener le sujet à un état de fonctionnement adapté ou à faciliter son accès à une chaîne de soins lorsque cela s'avère nécessaire » (p. 8). Pour eux, les objectifs d'une intervention de crise sont (a) la stabilisation $\mathrm{du}$ fonctionnement psychologique par la satisfaction des besoins physiques de base, puis l'abord des besoins psychologiques les plus fondamentaux ; (b) la réduction de la détresse liée au dysfonctionnement psychologique; (c) le retour à un fonctionnement psychologique tout à fait adapté ; et/ou (d) la facilitation de l'accès au niveau de soins suivant.

Roberts et coll. (2010) utilisent le terme d'intervention psychologique précoce pour les interventions commençant dans les trois mois suivant un événement traumatique, dans le but principalement d'empêcher un ESPT ou une détresse permanente chez des personnes souffrant de symptômes de stress traumatique, d'états de stress aigu (ESA) ou susceptibles de développer un ESPT ou d'autres troubles. D'autres auteurs (par exemple, Kehle et al., 2010) parlent de traitement psychologique précoce, intervention comprenant une psychothérapie et un traitement psychopharmacologique. En outre, le Ministère américain des anciens combattants (Veterans Affairs, VA) et le Ministère de la défense (Department of Defense, DoD) (2010) regroupent sous l'appellation de gestion $d u$ stress post-traumatique toutes les interventions de traitement du stress post-traumatique, de la réaction aiguë au stress (RAS), de l'ESA, de l'ESPT aigu et de l'ESPT chronique. (Cf. les directives de traitement du VA et du DoD [2010] pour les interventions actuellement recommandées et leurs limitations.) Comme on le voit dans ces exemples, le mot intervention peut faire référence à un large éventail d'activités allant de la satisfaction des besoins physiques immédiats à la psychopharmacothérapie.

Plusieurs études ont appliqué une approche dépistage/traitement à la suite de divers événements traumatiques afin d'identifier systématiquement ceux qui ont développé des symptômes post-traumatiques aigus et d'administrer un traitement le plus tôt possible (Brewin et al., 2008). Les Recommandations de bonnes pratiques cliniques pour la gestion $d u$ stress posttraumatique (publiées en 2010 par le VA et le DoD) mentionnaient que l'identification précoce d'un ESPT et son traitement rapide peuvent abréger la souffrance et réduire la gravité de la déficience fonctionnelle. Des interventions précoces brèves sont recommandées pour les patients présentant rapidement des niveaux de symptôme importants, de même que pour ceux qui sont handicapés par des symptômes psychologiques ou physiques aigus.
Différentes approches sont utilisées aujourd'hui pour traiter les traumas des premiers intervenants (par exemple, la psychoéducation, la gestion du stress consécutif à un incident critique, la thérapie cognitivecomportementale [TCC], la désensibilisation et le retraitement par les mouvements oculaires [EMDR]). Haugen et al. (2012) ont conclu que la littérature sur le traitement de l'ESPT chez les premiers intervenants n'était pas suffisamment fournie pour permettre des recommandations basées sur des preuves et suggéraient de mener des essais randomisés contrôlés (ERC) sur des traitements comme l'EMDR. La promotion de la santé mentale et du bien-être psychologique est reconnue comme une priorité générale de la recherche (p. ex. Organisation mondiale de la Santé [OMS], 2002).

\section{EMDR, premiers intervenants et intervention EMDR précoce}

L'EMDR est une approche de traitement intégrative en huit phases, qui s'appuie sur le modèle du traitement adaptatif de l'information (TAI) (F. Shapiro, 2001). Dans cette thérapie intégrative, les souvenirs dysfonctionnellement stockés sont considérés comme la première cause de la pathologie clinique, lorsque celle-ci n'est pas due à une déficience organique (F. Shapiro, 1989, 2001, 2007). L'EMDR se fonde sur l'hypothèse que le traitement de ces souvenirs conduit à leur résolution grâce à leur reconsolidation et leur assimilation au sein des réseaux plus grands de souvenirs adaptés (Oren \& Solomon, 2012). Il a été établi que l'EMDR était efficace dans le traitement de l'ESPT (American Psychiatric Association, 2004 ; Bisson \& Andrew, 2007) et est également utilisable pour traiter un large éventail d'autres souffrances cliniques provenant de l'expérience vécue (F. Shapiro, 2001; Solomon \& Shapiro, 2008). Les recommandations du VA/DoD (2010) classent l'EMDR en traitement de niveau " $A$ », c'est-à-dire une « forte recommandation de traiter par ce moyen les patients qui en relèvent » (p. 6).

Une revue de la littérature concernant le traitement de l'ESPT chronique chez les premiers intervenants a recensé des publications d'études de cas portant sur des officiers de police (Keenan \& Royle, 2007 ; Spates \& Burnette, 1995) et des pompiers (Kitchiner, 2004), et un ERC comparant le traitement EMDR et un programme classique de gestion du stress avec 62 officiers de police (Wilson, Tinker, Becker \& Logan, 2001).

On a aujourd'hui une grande expérience du travail clinique sur le terrain en intervention EMDR précoce (IEP) (Maxfield, 2008). Des résultats d'études publiées, il ressort que l'IEP est une intervention brève, avec 
des effets rapides, qui peut être utilisée sur le terrain ou dans les situations d'urgence.

Il existe un corpus de recherches soutenant l'utilisation de protocoles EMDR modifiés pour traiter les traumas aigus, en groupe ou en individuel (Jarero, Artigas \& Luber, 2011; E. Shapiro, 2012). La principale raison de ces modifications du protocole standard est que la consolidation du souvenir semble se modifier dans les semaines et les mois qui suivent un incident critique (F. Shapiro, 2001). Voir Elan Shapiro (2012) sur les progrès effectués dans le domaine de l'intervention psychologique précoce post-trauma en général et sur la place de l'intervention EMDR précoce en particulier.

Jarero et al. (2011) ont fait valoir que la thérapie EMDR d'intervention précoce a naturellement sa place en intervention de crise et dans le contexte de la chaîne des soins psychologiques lors d'une catastrophe, et qu'elle peut permettre l'intervention précoce parce que c'est une modalité brève de traitement. Les auteurs de cet article ont plaidé pour l'apport d'interventions de crise en fonction du déroulement des phases du rétablissement et de l'évaluation des besoins des victimes (Solomon, 2008).

\section{Usage paraprofessionnel du protocole EMDR individuel dans les situations traumatiques aiguës}

\section{Contexte}

Le protocole EMDR individuel pour un usage paraprofessionnel dans les situations de traumas graves (EMDR-PROPARA) fait partie d'un projet développé à l'initiative du Dr Francine Shapiro (communication personnelle, 28 juin 2012).

On sait que les pays en développement manquent souvent de ressources professionnelles pour répondre de façon adéquate aux grands événements traumatiques et que la prise en charge psychologique professionnelle du trauma peut y être insuffisante, voire inexistante. Cependant, ces pays disposent souvent d'un personnel paraprofessionnel formé, chargé de fournir interventions et soins. Il existe des antécédents de TCC délivrée efficacement par du personnel paraprofessionnel (praticiens de santé non mentale) avec des résultats comparables à ceux de praticiens professionnels (voir Hepner et al., 2012 ; Montgomery, Kunik, Wilson, Stanley \& Weiss, 2010).

Par conséquent, un programme spécial de formation en EMDR a été développé afin d'enseigner à des paraprofessionnels correctement sélectionnés, formés et supervisés, à faire de l'EMDR en groupe ou individuellement dans des situations traumatiques aiguës. La formation a commencé par un message du Dr Shapiro, transmis par les formateurs de l'atelier (des formateurs de formateurs de l'Institut EMDR). Le Dr Shapiro y mettait l'accent sur la sécurité du patient et encourageait le personnel paraprofessionnel à accorder une attention particulière au respect du traitement et à la rigueur des recherches, afin qu'on puisse rassembler les informations de façon systématique et les évaluer scientifiquement, pour en tirer des informations utiles.

Pour le format en groupe, on a choisi le " protocole EMDR intégratif de traitement en groupe " (Artigas, Jarero, Alcalá \& Lopez Cano, 2009). Ce protocole a été utilisé dans son format originel, ou adapté afin de correspondre à de nombreux cadres différents à travers le monde (Gelbach \& Davis, 2007 ; Maxfield, 2008). On en trouvera une vue d'ensemble dans l'article de Jarero et Artigas (2012).

L'EMDR-PROPARA a été développé par le premier auteur (IJ) pour la forme individuelle. Afin de s'assurer de la validité écologique du protocole (acceptabilité dans le monde réel; Brewer, 2000) une évaluation de terrain a été menée par des membres de groupes de travail de la Fondation latino-américaine et caraïbéenne pour la recherche sur les psychotraumatismes aux États-Unis, au Mexique, en Amérique Centrale, en Amérique du Sud et en Espagne. On a administré le traitement à différents publics en situation de trauma grave : des personnels militaires et des intervenants d'urgence en service, des civils (kidnapping) et des immigrants illégaux.

L'EMDR-PROPARA est une adaptation pour une utilisation paraprofessionnelle du protocole EMDR pour le traitement des incidents critiques récents (EMDR-PRECI). Ce dernier est une modification du "protocole pour le traitement des événements traumatiques récents » de F. Shapiro (2001), délivré sous forme de traitement individuel à des patients souffrant d'une traumatisation récente et permanente. Ce protocole a été développé sur le terrain afin de traiter des incidents critiques où des événements stressants corrélés entre eux se prolongent sur une grande période de temps et où il n'y a pas de période post-traumatique de sécurité permettant la consolidation du souvenir (voir Jarero et al., 2011, pour une description détaillée de ce protocole).

On a des preuves préliminaires de l'efficacité de l'EMDR-PRECI dans la réduction des symptômes de stress post-traumatique chez les adultes et le maintien de ces effets malgré la présence persistante de menaces et de danger dans le contexte d'une organisation de prise en charge psychologique en situation de catastrophe. L'EMDR-PRECI a été évaluée par 
Jarero et al. (2011) dans une étude qui comparait des groupes en traitement immédiat et des groupes de personnes sur liste d'attente, avec 18 adultes traumatisés par un récent tremblement de terre de magnitude 7,2 qui avait eu lieu en Basse-Californie, au Mexique. Les résultats montraient qu'une seule séance (durant entre 80 et 130 minutes) d'EMDR-PRECI produisait une amélioration significative des symptômes de stress post-traumatique pour le groupe en traitement immédiat comme pour le groupe en traitement retardé, avec des résultats qui s'étaient maintenus lors du suivi, douze semaines plus tard.

Une étude de terrain sur l'EMDR-PRECI a porté sur l'intervention précoce concernant des premiers intervenants mexicains traumatisés (policiers et personnel médico-légal) confrontés à des massacres et travaillant en situation de stress extrême et sans période de sécurité post-traumatique permettant la consolidation du souvenir (Jarero \& Uribe, 2011, 2012). Les résultats ont montré la diminution des scores autorapportés de stress post-traumatique et de symptômes d'ESPT, confortant l'idée que l'EMDR peut être utilisée efficacement comme intervention précoce pour les premiers intervenants souffrant de traumatismes. Il est apparu que l'EMDR-PRECI aidait à prévenir l'apparition d'un ESPT chronique, et à augmenter la résilience psychologique et émotionnelle des personnels.

Développement de l'EMDR-PROPARA. Norris, Murphy, Baker et Perilla (2004) souhaitent « des interventions précoces et durables qui apportent des soins psychologiques aux victimes de catastrophe d'une manière qui soit culturellement appropriée et réalisable dans des lieux où on trouve peu de professionnels de la santé mentale sur lesquels s'appuyer » (pp. 290-291). L'EMDR-PROPARA a été conçu pour une utilisation paraprofessionnelle. Il vise à réduire la gravité des symptômes de stress post-traumatique ainsi que les symptômes de détresse somatique, les difficultés de gestion du stress et les difficultés d'ordre professionnel, familial et social. Il a été spécifiquement conçu pour répondre aux trois présentations cliniques qui sont courantes chez les personnes qui ne parviennent pas à se remettre d'une situation traumatique aiguë : variantes infracliniques de l'état de stress aigu (ESA) ou de l'ESPT aigu, (b) difficultés fonctionnelles et/ou sociales et (c) symptômes infracliniques de stress traumatique ayant un impact sur leur qualité de vie. Ces présentations peuvent se développer au cours du temps ou à la suite d'interventions précoces (c'est-à-dire après une prise en charge des besoins physiques immédiats, à la suite d'un soutien d'ordre social ou spirituel, d'une psychoéducation, d'une normalisation ou de premiers soins d'ordre psychologique). Dans l'expérience clinique et de terrain des auteurs, ces présentations pourraient provenir des trois situations de trauma aigu mentionnées au début de cet article : le syndrome de stress aigu, l'événement récent, et le réseau de souvenirs d'expositions cumulatives au trauma.

Dans la mesure où l'application de l'EMDR par des paraprofessionnels est un nouveau projet, nous considérons qu'un suivi permettant de déterminer l'état $\mathrm{du}$ patient après l'intervention EMDR-PROPARA est indispensable. Nous recommandons le recours à une liste de contrôle validée, auto-administrée (par exemple, le SPRINT), pour garantir un examen systématique, standardisé et efficace des symptômes du patient et de son passé d'exposition traumatique. L'utilisation régulière et systématique de ces listes de contrôle permettrait l'évaluation des réponses au traitement et les progrès du patient $(\mathrm{VA} / \mathrm{DoD}, 2010)$. La qualité des soins fournis par le paraprofessionnel, et le paraprofessionnel lui-même, doivent être contrôlés avec soin par une supervision, dans un contexte positif, non critique.

Les patients qui n'obtiennent pas d'amélioration ou dont l'état empire doivent être orientés vers une thérapie fondée sur des preuves (par exemple, l'EMDR ou la TCC centrée sur le trauma). Dans les régions où on trouve peu ou pas de prise en charge professionnelle du trauma psychologique, on envisagera l'utilisation d'un traitement d'appoint avec des modalités de soins alternatives, telles qu'une médecine complémentaire proposant des traitements corps-esprit (p. ex. acupuncture, méditation, yoga, relaxation), en cohérence avec les ressources disponibles et avec les systèmes de croyances du patient (Strauss \& Lang, 2012).

\section{Méthode}

La commission d'examen de la Fondation latinoaméricaine et caraibéenne pour la recherche sur les psychotraumatismes a approuvé le protocole de recherche. Les participants ont donné par écrit leur consentement éclairé avant leur inscription. Les recherches avaient pour objectif d'évaluer l'efficacité de l'EMDR-PROPARA sur un événement traumatique récent. En conséquence, lors de l'évaluation et du traitement, on a demandé à tous les participants de se concentrer sur leur pire expérience de travail au cours des trois derniers mois. Afin de mesurer l'impact de l'EMDR-PROPARA, on a réalisé un protocole pré/ post dans le but de comparer l'EMDR-PROPARA et la thérapie de soutien. Des mesures relevées à quatre occasions permettaient l'exploration des effets du traitement à plus long terme. 


\section{Les participants}

Trente-neuf premiers intervenants en service actif (20 hommes, 19 femmes) de Sonora, au Mexique, ont été recrutés sur la base du volontariat parmi des ambulanciers de la Croix-Rouge $(n=15)$, des opérateurs de ligne téléphonique d'urgence $(n=15)$ et des pompiers $(n=9)$. Les participants ont été répartis de façon aléatoire pour recevoir, soit la thérapie de soutien $(n=20)$, soit l'EMDR-PROPARA $(n=19)$. Les critères d'inclusion étaient les suivants : (a) être des premiers intervenants, (b) être en service actif et (c) avoir entre 18 et 60 ans. Les critères d'exclusion étaient les suivants : (a) idées suicidaires au moment de l'étude, (b) diagnostic de trouble psychotique ou bipolaire, de trouble mental organique ou de toxicomanie, (c) idées suicidaires ou d'homicide au moment de l'étude et (d) difficultés cognitives importantes. Tous les participants ont continué d'être en service actif pendant la durée de l'étude. Leur présence au traitement n'était pas demandée par leur employeur et il n'y a eu aucun abandon au cours de cette étude.

\section{Les mesures}

Le SPRINT est un questionnaire d'entretien ou d'autoévaluation en huit items, ayant des propriétés psychométriques robustes, qui peut servir de mesure fiable, valide et homogène de la gravité de l'ESPT et de son amélioration générale, de même qu'un moyen de mesure de la détresse physique, de la capacité à faire face au stress et des difficultés d'ordre professionnel, familial ou social. Chaque item du SPRINT est évalué sur une échelle en 5 points : pas du tout (0), un petit peu (1), modérément (2), passablement (3), beaucoup (4). Des scores entre 18 et 32 correspondent à des symptômes marqués ou graves d'ESPT, entre 11 et 17 à des symptômes modérés, de 7 à 10 à des symptômes légers et des scores inférieurs ou égaux à 6 indiquent peu ou pas de symptômes. Le SPRINT contient également deux items additionnels permettant de mesurer l'amélioration générale selon une variation de pourcentage et par un indice de gravité. Ce questionnaire a été traduit de l'anglais à l'espagnol puis, de nouveau, de l'espagnol à l'anglais et revu et autorisé par un de ses auteurs. Le SPRINT fonctionne de manière similaire à la Clinical-Administered PTSD Scale (CAPS) dans l'évaluation des groupes de symptômes et des scores totaux et peut être utilisé comme instrument diagnostique (Vaishnavi, Payne, Connor \& Davidson, 2006). Au SPRINT, un score seuil (cutoff score) de 14 ou plus possède une sensibilité de $95 \%$ de détection d'un ESPT et une spécificité de $96 \%$ dans l'exclusion de ce diagnostic, avec une précision globale d'assignement correct de $96 \%$ (Connor \& Davidson, 2001).

\section{Procédure}

Le recrutement s'est déroulé du 6 au 30 août 2012, en deux étapes : (a) les chercheurs rencontraient les participants potentiels dans leurs institutions et expliquaient l'étude avec ses critères d'inclusion et d'exclusion et (b) les volontaires donnaient par écrit leur consentement éclairé. Des informations démographiques étaient également recueillies.

Les participants étaient évalués avec le SPRINT avant le traitement (temps 1), après le traitement (temps 2), un mois après le traitement (temps 3) et trois mois après le traitement (temps 4). Toutes les évaluations post-traitement et de suivi à un et trois mois ont été conduites par un professionnel n'ayant pas connaissance du statut des groupes de traitement.

\section{Traitements}

Les participants ont été répartis de façon aléatoire en deux groupes recevant soit une thérapie de soutien $(n=20)$ soit l'EMDR-PROPARA $(n=19)$. Chaque groupe a reçu des séances individuelles d'une heure et demie conduites par un des quatre cliniciens EMDR de l'étude (un formateur de formateurs de l'institut EMDR, un superviseur EMDR approuvé et deux thérapeutes EMDR certifiés). Les quatre thérapeutes EMDR avaient été formés à l'EMDR-PROPARA par le premier auteur. La conformité du traitement était facilitée par un strict respect des protocoles thérapeutiques.

L'EMDR-PROPARA. L'EMDR-PROPARA comprend les phases suivantes :

1. Préparation de l'espace de travail.

2. Historique : (a) collecte d'informations démographiques, (b) questions concernant l'incident critique, (c) évaluation des symptômes physiques, mentaux, émotionnels et comportementaux et (d) administration de l'instrument.

3. Préparation du participant : techniques d'autoapaisement.

4. Facilitation du traitement du souvenir traumatique : (a) évaluation du souvenir traumatique, (b) désensibilisation, (c) installation ou renforcement d'une opinion positive de soi et (d) scanner corporel.

5. Clôture de la séance.

6. Informations concernant la gestion du stress.

7. Réévaluation : (a) évaluation de la séance précédente et (b) redémarrage d'une séance.

8. Réévaluation avant fin du traitement : (a) stimuli du présent, (b) modèle futur, (c) résolution des symptômes, (d) progression de la personne après le trauma et (e) administration de l'instrument. 
La principale différence entre l'EMDR-PRECI et l'EMDR-PROPARA porte sur les stimulations bilatérales (SBL) pendant le traitement du souvenir traumatique. Dans l'EMDR-PRECI, les mouvements oculaires sont la première option de SBA. Dans l'EMDR-PROPARA, le " papillon » est la seule méthode utilisée. Artigas et Jarero (à paraître) estiment que le contrôle que donne aux patients le fait de " faire le papillon » les aide à conserver une sensation de sécurité pendant le traitement des souvenirs traumatiques. Les auteurs supposent que lors des phases de papillon/SBA, le système TAI (Shapiro, 2001) régule la stimulation afin de maintenir les patients dans leur fenêtre de tolérance (Corrigan, Fisher $\&$ Nutt, 2011) et de permettre un retraitement approprié. Selon Shapiro, en effet, le système intrinsèque de traitement de l'information du patient ainsi que ses réseaux de souvenirs associés sont les moyens les plus efficaces pour obtenir des effets cliniques optimaux.

La thérapie de soutien. Les séances de thérapie de soutien comprenaient l'apport d'informations sur le traumatisme, l'acquisition de compétences de résolution de problèmes et l'apport d'un soutien inconditionnel de la part du thérapeute. La thérapie de soutien évitait spécifiquement les techniques d'exposition ou de gestion de l'anxiété. Cette technique psychologique a été sélectionnée pour cette comparaison, parce qu'elle a été utilisée à de nombreuses reprises dans les essais randomisés contrôlés évaluant l'efficacité de la TCC centrée sur le trauma en tant qu'intervention d'urgence dans le traitement des symptômes de stress traumatique aigu (par exemple, Bryant, Harvey, Sackville, Dang \& Basten, 1998 ; Bryant, Sackville, Dang, Moulds \& Guthrie, 1999 ; Foa, Rothbaum, Riggs \& Murdock, 1991 ; Roberts et al., 2010)

\section{Analyse statistique}

Étant donné la conception de l'étude, nous avons construit un modèle linéaire général (MLG) de mesures répétées afin d'évaluer les effets de l'EMDRPROPARA sur les scores au SPRINT. Les deux raisons essentielles de l'utilisation de mesures répétées étaient (a) d'augmenter la puissance statistique et (B) de connaître les effets du traitement sur la durée. En plus du MLG, des tests $t$ ont été conduits afin de comparer les scores à chaque temps de l'évaluation.

\section{Résultats}

Les données montrent clairement les effets de l'EMDR-PROPARA : réduction des symptômes de stress post-traumatique des participants et maintien à long terme d'une diminution de ces symptômes, en dépit de leur exposition permanente, en raison de leur activité professionnelle, à des événements stressants similaires.

Les participants ont été évalués au moyen du SPRINT à quatre occasions : avant traitement (temps 1), post-traitement (temps 2), au cours du suivi un mois plus tard (temps 3), lors du suivi trois mois plus tard (temps 4). La Figure 1 montre l'importante différence globale des résultats entre la thérapie de soutien et l'EMDR-PROPARA. Le Tableau 1 montre les scores moyens et les écarts-types pour les deux groupes au cours des quatre SPRINT.

\section{Équivalence des groupes avant traitement}

Les scores moyens au SPRINT pour les participants (EMDR-PROPARA : $\chi^{2}=17,26 ; \sigma=4,13$; thérapie de soutien : $\left.\chi^{2}=17,70 ; \sigma=4,93\right)$ étaient dans la zone modérée et au-dessus du score seuil de 14 indiquant l'ESPT (Tableau 1). Il n'y avait pas de différence significative entre les scores initiaux des deux groupes de traitement $: t(37)=0,299 ; p=0,76$.

\section{Effets du traitement au cours du temps}

Les résultats des mesures répétées MLG ont mis en lumière une différence générale significative entre les groupes de thérapie de soutien et d'EMDR-PROPARA au fil du temps, $F(1,35)=138,97 ; p=0,001$. Ces résultats confirment les principaux effets de groupe et les principaux effets du temps au fil des quatre mesures. Les effets entre sujets ont montré des différences entre les groupes de la thérapie de soutien et d'EMDR-PROPARA, $F(1,35)=92,29 ; p=.0,001$. L'interaction entre sexe et groupe n'a montré aucune différence significative, $F(1,35)=1,260 ; p=269$, indiquant que les hommes et les femmes ont le même pattern de réponse. La comparaison intrasujets a montré une interaction importante de la durée et des groupes, $F(1,35)=524,87 ; p=0,001$. Cet effet est visible sur la Figure 1 qui illustre la diminution constante des scores moyens du groupe EMDR-PROPARA.

\section{Effet du traitement à court terme}

Un test $t$ pour échantillons indépendants a été utilisé afin de comparer les scores post-traitement au SPRINT pour l'EMDR-PROPARA et la thérapie de soutien. Le score au SPRINT du groupe EMDRPROPARA était significativement plus bas que celui du groupe de thérapie de soutien, $t(37)=6,35$; $p=0,001$ (Tableau 1). Ces résultats montrent l'efficacité immédiate de l'EMDR-PROPARA. 


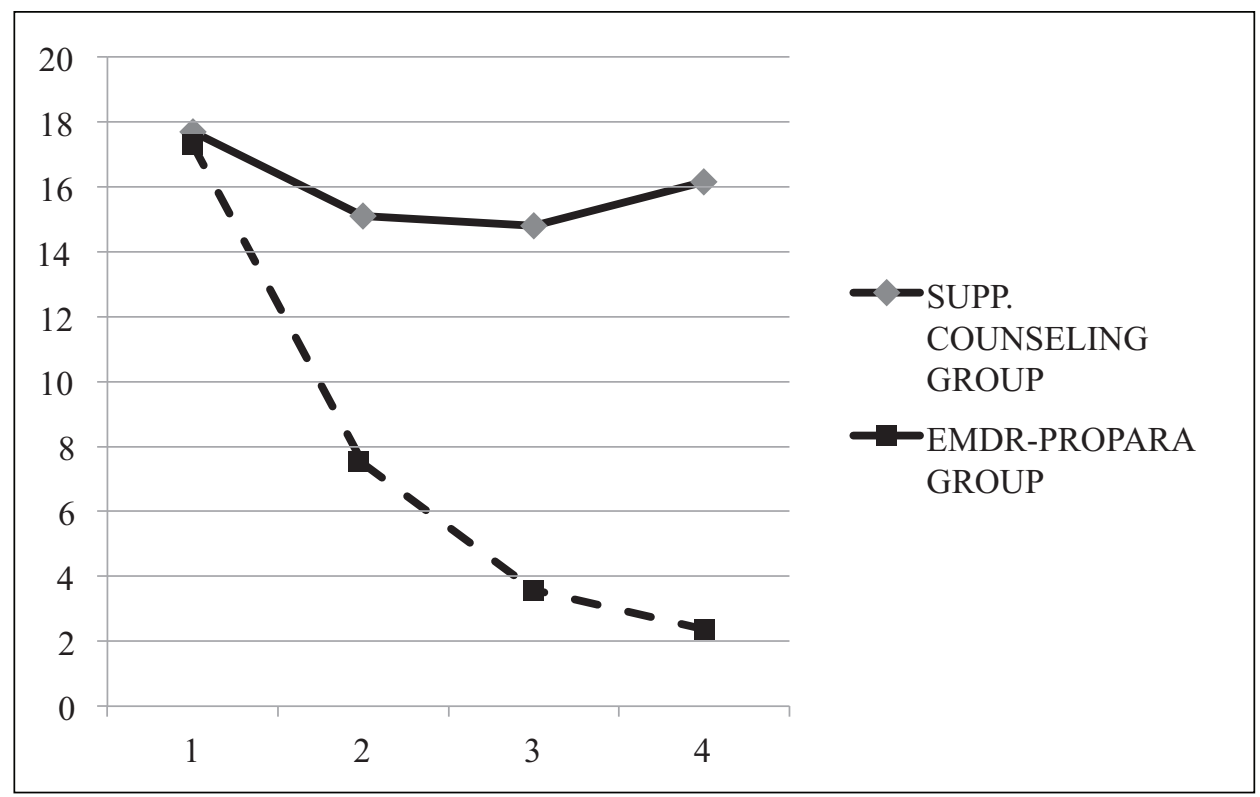

FIGURA 1. Scores moyens au Short PTSD Rating Interview (SPRINT). Temps $1=$ avant traitement, temps $2=$ après traitement, temps $3=$ suivi à un mois, et temps $4=$ suivi à trois mois. EMDR-PROPARA $=$ eye movement desensitization and reprocessing individual protocol for paraprofessional use in acute trauma situations (protocole individuel de désensibilisation et de retraitement pour un usage paraprofessionnel dans les situations de trauma aigu).

Note. Supp. counseling group $=$ groupe de thérapie de soutien $;$ EMDR-PROPARA group $=$ groupe EMDR-PROPARA.

\section{Maintien des effets du traitement}

Une analyse statistique utilisant un test $t$ pour échantillons indépendants a comparé les scores au SPRINT de l'EMDR-PROPARA et de la thérapie de soutien pour chacune des deux évaluations de suivi (Tableau 1). À chaque fois, comme on s'y attendait, la moyenne du groupe EMDR-PROPARA était significativement plus basse que celle du groupe de thérapie de soutien.

TABLEAU 1. Moyennes (écarts-types) et comparaisons statistiques des scores au SPRINT

\begin{tabular}{|c|c|c|c|c|}
\hline \multirow[b]{2}{*}{ Temps } & \multicolumn{2}{|c|}{ Moyenne (écart-type) } & \multicolumn{2}{|c|}{$\begin{array}{c}\text { Comparaison } \\
\text { statistique }\end{array}$} \\
\hline & $\begin{array}{l}\text { Thérapie } \\
\text { de soutien } \\
(n=20)\end{array}$ & $\begin{array}{c}\text { EMDR- } \\
\text { PROPARA } \\
(n=19)\end{array}$ & $t(37)$ & $p$ \\
\hline Prétraitement & $17,70(4,94)$ & $17,26(4,41)$ & 0,299 & 0,767 \\
\hline Post-traitement & $15,10(4,59)$ & $7,47(2,54)$ & 6,35 & 0,001 \\
\hline Premier suivi & $14,80(5,34)$ & $3,58(1,61)$ & 8,77 & 0,001 \\
\hline Second suivi & $16,15(3,92)$ & $2,36(0,83)$ & 14,98 & 0,001 \\
\hline
\end{tabular}

Note. SPRINT $=$ Short PTSD Rating Interview ; EMDR-PROPARA $=$ EMDR individual protocol for paraprofessional use in acute trauma situations $=$ protocole individuel de désensibilisation et de retraitement pour un usage paraprofessionnel dans les situations de trauma aigu.

\section{Amélioration globale subjective}

Le SPRINT contient deux items permettant de mesurer l'amélioration générale. L'item 1 évalue le changement de pourcentage par l'interrogation : "Définissez à quel point vous vous sentez mieux depuis que le traitement a commencé ? Donnez un pourcentage entre 0 et 100 . " Le point 2 évalue la gravité des symptômes en demandant au participant d'utiliser l'échelle en 5 points de Likert (où $1=$ pire, $2=$ pas de changement, $3=$ un changement minime, $4=$ un changement important et $5=$ un changement très important). On l'introduit par l'interrogation, " à quel point vos symptômes se sont-ils améliorés depuis le début du traitement ? " Pour l'item 1, la réponse moyenne, lors du suivi, pour le groupe EMDR-PROPARA était de $91 \%$; et pour l'item 2, la réponse moyenne lors du suivi était un changement très important. Inversement, pour l'item 1, la réponse moyenne lors du suivi pour le groupe de thérapie de soutien était de $63 \%$ et à l'item 2 , la réponse moyenne était un changement minime.

\section{Discussion}

Cette étude visait à examiner l'efficacité de l'EMDRPROPARA, un protocole de traitement individuel conçu pour une utilisation paraprofessionnelle dans les situations de traumas aigu. Les participants du 
groupe EMDR-PROPARA ont présenté des améliorations immédiatement après le traitement, et leurs scores au SPRINT ont montré d'autres baisses au cours du second suivi, trois mois après le traitement. En comparaison, les contrôles de la thérapie de soutien enregistraient une diminution non significative post-traitement et une augmentation des scores du SPRINT lors du second suivi. Les résultats fournissent donc un soutien préliminaire en faveur de l'EMDRPROPARA pour son efficacité à réduire la gravité des symptômes post-traumatiques et à améliorer l'état global subjectif.

Au départ, le score moyen des deux groupes de traitement était supérieur à 14 , score considéré comme le seuil indiquant un ESPT. Les scores du groupe en thérapie de soutien, au cours des quatre mesures, ne sont jamais descendus en dessous de 14, alors que dans le groupe EMDR-PROPARA, le score moyen au cours de l'évaluation post-traitement était de 7,47.

Les données recueillies au cours des mesures de suivi ont confirmé que les participants des deux groupes ont poursuivi leur activité de travail de la même manière qu'avant le traitement. Aucune modification significative dans les conditions de vie des participants n’a été signalée pendant l'étude. Par conséquent, les résultats de l'étude ne peuvent pas être attribués à des changements dans l'environnement de travail ou à d'autres événements stressants. De plus, aucun problème psychologique préexistant, aucun autre facteur de risque rendant les sujets plus vulnérables à un ESPT et pouvant expliquer les différences entre les deux groupes n’ont été trouvés.

\section{Effets à long terme du trauma et bénéfices du traitement}

Les différences entre l'EMDR-PROPARA et la thérapie de soutien se sont maintenues tout au long de l'étude à partir des séances de traitement. Ce pattern est cohérent avec les recherches portant sur l'effet longitudinal du traitement EMDR chez des sujets exposés en permanence à des événements stressants en raison de leurs conditions de travail (Jarero \& Uribe, 2012) et qui sont, par conséquent, davantage susceptibles de développer un ESPT chronique (Orcutt, Erickson \& Wolfe, 2004).

L'intervention EMDR-PROPARA pourrait donc être un facteur clé de protection des premiers intervenants contre l'apparition d'une psychopathologie. Elle constituerait un traitement rapide, réduisant de façon efficace les symptômes aigus, de telle sorte que des traitements plus intensifs ou difficiles d'accès (tels que l'exposition prolongée ou un traitement pharmacologique) ne seraient plus nécessaires. Dans des situations où le nombre de victimes souffrant de trauma est élevé et où le nombre de cliniciens expérimentés dans le trauma est limité, l'EMDR-PROPARA pourrait également fonctionner comme un système de triage permettant d'orienter les efforts thérapeutiques les plus importants seulement vers les patients résistants, qui sont plus susceptibles de développer des syndromes chroniques.

Dans la mesure où la communauté compte sur les services des premiers intervenants en temps de crise, les recherches sur le trauma au sein de cette population spécifique deviennent d'une importance cruciale. Nous avons suivi les recommandations de Roberts et al. (2010), qui préconisaient des recherches permettant d'identifier les moyens les plus efficaces d'aide psychologique dans les premiers moments après un événement traumatique.

\section{Références}

American Psychiatric Association. (2004). Practice guidelines for the treatment of patients with acute stress disorder and posttraumatic stress disorder. Arlington, VA: Author.

Artigas, L., \& Jarero, I. (2013). The butterfly hug. In M. Luber (Ed.), Implementing EMDR early mental health interventions for man-made and natural disasters: Models, scripted protocols and summary sheets. New York, NY : Springer.

Artigas, L., Jarero, I., Alcalá, N., \& Lopez Cano, T. (2009). The EMDR Integrative Group Treatment Protocol (IGTP). In M. Luber (Ed.), Eye movement desensitization and reprocessing (EMDR) scripted protocols: Basic and special situations (pp. 279-288). New York, NY : Springer.

Benedek, D. M., Fullerton, C., \& Ursano, R. J. (2007). First responders: Mental health consequences of natural and human-made disasters for public health and public safety workers. Annual Review of Psychology, 28, 55-68.

Bisson, J., \& Andrew, M. (2007). Psychological treatment of post-traumatic stress disorder (PTSD). Cochrane Database of Systematic Reviews, 18(3), CD003388. http:// dx.doi.org/10.1002/14651858.CD003388.pub3

Bisson, J. I., Roberts, N., \& Macho, G. S. (2003). Service innovations : The Cardiff traumatic stress initiative: An evidence based approach to early psychological intervention following traumatic events. Psychiatric Bulletin, 27(4), 145-147.

Brewer, M. B. (2000). Research design and issues of validity. In H. T. Reis \& C. M. Juss (Eds.), Handbook of research methods in social and personality psychology (pp. 3-15). Cambridge, United Kingdom: Cambridge University Press.

Brewin, C. R., Scragg, P., Robertson, M., Thompson, M., d'Ardenne, P., \& Ehlers, A. (2008). Promoting mental health following the London bombings: A screen and treat approach. Journal of Traumatic Stress, 21, 3-8. 
Bryant, R. A., Harvey, A. G., Sackville, T., Dang, S. T., \& Basten, C. (1998). Treatment of acute stress disorder: A comparison of cognitive behavioural therapy and supportive counseling. Journal Consult Clinical Psychology, 66, 862-866.

Bryant, R. A., Sackville, T., Dang, S. T., Moulds, M., \& Guthrie, R. (1999). Treating acute stress disorder: An evaluation of cognitive behavioural therapy and supportive counseling techniques. American Journal of Psychiatry, 156, 1780-1786.

Connor, K. M., \& Davidson, J. R. T. (2001). SPRINT : A brief global assessment of post-traumatic stress disorder. International Clinical Psychopharmacology, 16(5), 279-284.

Corrigan, F. M., Fisher, J. J., \& Nutt, D. J. (2011). Autonomic dysregulation and the Window of Tolerance model of the effects of complex emotional trauma. Journal of Psychopharmacology, 25(1), 17-25.

Creamer, M. C., Varker, T., Bisson, J., Darte, K., Greenberg, N., Lau, W., Forbes, D. (2012). Guidelines for peer support in high-risk organizations: An international consensus study using the Delphi method. Journal of Traumatic Stress, 25, 134-141

Everly, G. S., Jr., \& Mitchell, J. T. (2008). Integrative crisis intervention and disaster mental health. Ellicott City, MD : Chevron.

Figley, C. E. (1995). Compassion fatigue : Coping with secondary stress in those who treat the traumatized. New York, NY : Brunner/Mazel.

Foa, E. B., Rothbaum, B. O., Riggs, D. S., \& Murdock, T. B. (1991). Treatment of posttraumatic stress disorder in rape victims: A comparison between cognitive-behavioural procedures and counseling. Journal Consult Clinical Psychology, 59, 715-723.

Gelbach, R., \& Davis, K. (2007). Disaster response: EMDR and family systems therapy under communitywide stress. In F. Shapiro, F.W. Kaslow, \& L. Maxfield (Eds.), Handbook of EMDR and family therapy processes (pp. 387-406). New York, NY : John Wiley \& Sons.

Guo, U., Chen, C., Lu, M., Tan, H. K., Lee, H., \& Wang, T. (2004). Posttraumatic stress disorder among professional and nonprofessional rescuers involved in an earthquake in Taiwan. Psychiatry Research, 127, 35-41.

Haugen, P. T., Evces, M., \& Weiss, D. S. (2012). Treating posttraumatic stress disorder in first responders: A systematic review. Clinical Psychology Review, 32, 370-380.

Hepner, K. A., Miranda, J., Woo, S., Watkins, K. E., Lagomasino, I., Wiseman, S. H., \& Munoz, R. F. (2012). Building recovery by improving goals, habits, and thoughts (BRIGHT): A group cognitive behavioral therapy for depression in clients with co-occurring alcohol and drug use problems. Group member's workbook. Santa Monica, CA : RAND Corporation. Téléchargé depuis http://www .rand. org/pubs/technical_reports/TR977z2

Jarero, I., \& Artigas, L. (2012). The EMDR Integrative Group Treatment Protocol: EMDR group treatment for early intervention following critical incidents. European Review of Applied Psychology, 62, 219-222.

Jarero, I., Artigas, L., \& Luber, M. (2011). The EMDR protocol for recent critical incidents: Application in a disaster mental health continuum of care context. Journal of EMDR Practice and Research, 5(3), 82-94.

Jarero, I., \& Uribe, S. (2011). The EMDR protocol for recent critical incidents: Brief report of an application in a human massacre situation. Journal of EMDR Practice and Research, 5(4), 156-165.

Jarero, I., \& Uribe, S. (2012). The EMDR protocol for recent critical incidents: Follow-up report of an application in a human massacre situation. Journal of EMDR Practice and Research, 6(2), 50-61.

Keenan, P., \& Royle, I. (2007). Vicarious trauma and first responders: A case study utilizing eye movement desensitization and reprocessing (EMDR) as the primary treatment modality. International Journal of Emergency Mental Health, 9, 291-298.

Kehle, S. M., Polusny, M. A., Murdoch, M., Erbes, C., Arbisi, P., Thuras, P., \& Meis, L. (2010). Early mental health treatment-seeking among U.S. National Guard soldiers deployed to Iraq. Journal of Traumatic Stress, 23(1), 33-40.

Kitchiner, N. J. (2004). Psychological treatment of three urban firefighters with posttraumatic stress disorder using eye movement desensitization and reprocessing (EMDR) therapy. Complementary Therapies in Nursing \& Midwifery, 10, 186-193.

Kleim, B., \& Westphal, M., (2011). Mental health in first responders: A review and recommendation for prevention and intervention strategies. Traumatology, 17(4), 17-24.

Maxfield, L. (2008). EMDR treatment of recent events and community disasters. Journal of EMDR Practice \& $R e$ search, 2(2), 74-78.

Montgomery, E. C., Kunik, M. E., Wilson, N., Stanley, M. A., \& Weiss, B. (2010). Can paraprofessionals deliver cognitive-behavioral therapy to treat anxiety and depressive symptoms ? Bulletin of the Menninger Clinic, 74(1), 45-62.

Norris, F. H., Murphy, A. D., Baker, C. K., \& Perilla, J. L. (2004). Postdisaster PTSD over four waves of a panel study of Mexico's 1999 flood. Journal of Traumatic Stress, 17(4), 283-292.

Orcutt, H. K., Erickson, D. J., \& Wolfe, J. (2004). The course of PTSD symptoms among Gulf War veterans: A growth mixture modeling approach. Journal of Traumatic Stress, 17(3), 195-202.

Oren, E., \& Solomon, R. (2012). EMDR therapy: An overview of its development and mechanisms of action. European Review of Applied Psychology, 62, 197-203.

Peñalba, V., McGuire, H., \& Leite, J. R. (2008). Psychosocial interventions for prevention of psychological disorders in law enforcement officers. Cochrane Database of Systematic Reviews, (3), CD005601.

Roberts, N. P., Kitchiner, N. J., Kenardy, J., \& Bisson, J. I. (2010). Early psychological interventions to treat acute 
traumatic stress symptoms. Cochrane Database of Systematic Reviews, (3), CD007944.

Scully, P. J. (2011). Taking care of staff: A comprehensive model of support for paramedic and emergency medical dispatchers. Traumatology, 17(4), 35-42.

Shakespeare-Finch, J. (2011). Primary and secondary trauma in emergency personnel. Traumatology, 17(4), 1-2.

Shapiro, E. (2012). EMDR and early psychological intervention following trauma. European Review of Applied Psycho$\log y, 62,241-251$.

Shapiro, F. (1989). Efficacy of the eye movement desensitization procedure in the treatment of traumatic memories. Journal of Traumatic Stress Studies, 2, 199-223.

Shapiro, F. (2001). Eye movement desensitization and reprocessing: Basic principles, protocols, and procedures (2e éd.). New York, NY : Guilford Press.

Shapiro, F. (2007). EMDR, adaptive information processing, and case conceptualization. Journal of EMDR Practice and Research, 1, 68-87.

Solomon, R. (2008). Critical incident interventions. Journal of EMDR Practice and Research, 2, 160-165.

Solomon, R. M., \& Shapiro, F. (2008). EMDR and the adaptive information processing model: Potential mechanism of change. Journal of EMDR Practice and Research, 2(4), 315-325.

Spates, C. R., \& Burnette, M. M. (1995). Eye movement desensitization: Three unusual cases. Journal of Behavior Therapy and Treatment Psychiatry, 26, 321-329.

Strauss, J. L., \& Lang, A. J. (2012). Complementary and alternative treatments for PTSD. PTSD Research Quarterly, 23(2), 1-7.

U.S. Department of Veterans Affairs and Department of Defense. (2010). VA/DoD clinical practice guidelines for management of post-traumatic stress. Version 2.0.
Téléchargé depuis http:/ / www.healthquality. va.gov/ ptsd/CPG_Summary_FINAL_MgmtofPTSD final.pdf

Vaishnavi, S., Payne, V., Connor, K., \& Davidson, J. R. (2006). A comparison of the SPRINT and CAPS assessment scales for posttraumatic stress disorder. Depression and Anxiety, 23(7), 437-440.

Van der Kolk, B. A., \& Van der Hart, O. (1991). The intrusive past: The flexibility of memory and the engraving of trauma. American Imago, 48(4), 425-454.

Wilson, S. A., Tinker, R. H., Becker, L. A., \& Logan, C. R. (2001). Stress management with law enforcement personnel : A controlled outcome study of EMDR versus a traditional stress management program. International Journal of Stress Management, 8, 179-200.

World Health Organization (2002). The World Health Report 2002: Reducing risks, promoting healthy life. Geneva, Switzerland: Author.

Remerciements. Nos remerciements vont au Dr Francine Shapiro pour ses inestimables conseils et son soutien ; aux membres de l'équipe qui a testé la validité écologique du protocole sur le terrain : Carolina Amaya, María Alicia Cavazos, Martha Givaudan, Cristina Jarero, Alaide Miranda, Shaila Romero, Georgina Sánchez, Susana Uribe, et Dinorah Villalobos ; à Carolina Amaya et Monica Ponzanelli pour leur recensement des études portant sur les ambulanciers et les policiers, et à Cristina Jarero pour son assistance sur les études en langue anglaise.

Merci d'adresser toute correspondance concernant cet article à Ignacio Jarero, PhD, EdD, Boulevard de la Luz 771, Jardines del Pedregal, Álvaro Obregón, México City 01900, Mexique. Courriel : nacho@amamecrisis.com.mx 